\title{
Some Weak Fragments of $H A$ and Certain Closure Properties
}

\author{
Morteza Moniri and Mojtaba Moniri
}

\begin{abstract}
We show that Intuitionistic Open Induction iop is not closed under the rule $D N S\left(\exists_{1}^{-}\right)$. This is established by constructing a Kripke model of $i o p+\neg L_{y}(2 y>x)$, where $L_{y}(2 y>x)$ is universally quantified on $x$. On the other hand, we prove that iop is equivalent with the intuitionistic theory axiomatized by $P A^{-}$plus the scheme of weak $\neg \neg L N P$ for open formulas, where universal quantification on the parameters precedes double negation. We also show that for any open formula $\varphi(y)$ having only $y$ free, $\left(P A^{-}\right)^{i} \vdash L_{y} \varphi(y)$. We observe that the theories $i o p, i \forall_{1}$ and $i \Pi_{1}$ are closed under Friedman's translation by negated formulas and so under $V R$ and $I P$. We include some remarks on the classical worlds in Kripke models of iop.
\end{abstract}

2000 Mathematics Subject Classification: 03F30, 03F55, 03H15.

Key words and phrases: Shepherdson's Model, Intuitionistic Open Induction, Intuitionistic Open Least Number Principle, Double Negation Shift, Friedman's Translation, Pruning, Visser's Rule, Independence of Premises.

This work took place while the first author was a $\mathrm{PhD}$ student at Institute for Studies in Theoretical Physics and Mathematics (IPM), Tehran, Iran. Both authors acknowledge IPM's support.

\section{Preliminaries}

1.1 Let $D O R$ (resp. $P A^{-}$) be the finite set of usual axioms (including Trichotomy) for discretely ordered commutative rings with 1 (resp. their nonnegative parts) in the language $L=\{+, \cdot,<$ $, 0,1\}$ of arithmetic. Peano Arithmetic $P A$ (resp. Heyting Arithmetic $H A$ ) is the classical (resp. intuitionistic, obtained by dropping the principle $P E M$ of excluded middle whose instance $P E M_{\varphi}$ on a formula $\varphi$ is $\varphi \vee \neg \varphi$ ) first order theory axiomatized by $P A^{-}$together with the induction scheme whose instance with respect to a distinguished free variable $x$ on a formula $\varphi(x, \bar{y})$ is

$$
I_{x} \varphi=I_{x} \varphi(x, \bar{y}): \forall \bar{y}(\varphi(0, \bar{y}) \wedge \forall x(\varphi(x, \bar{y}) \rightarrow \varphi(x+1, \bar{y})) \rightarrow \forall x \varphi(x, \bar{y})) .
$$


1.2 The classical Open Induction fragment Iop of $P A$ is axiomatized by only keeping (besides $P A^{-}$) the instances of induction on open, i.e. quantifier-free, formulas. It was first studied by Shepherdson [Sh]. He constructed a (recursive) nonstandard model proving independence results, such as irrationality of $\sqrt{2}$, from Iop. Let $\tilde{\mathbb{Q}}$ be the field of real algebraic numbers. Shepherdson's model was

$$
\begin{gathered}
\mathcal{S}_{t}(\mathbb{N})=\cup_{n \in \mathbb{Z}>0}\left(t^{\frac{1}{n}} \tilde{\mathbb{Q}}\left[t^{\frac{1}{n}}\right]+\mathbb{Z}\right)^{\geq 0}=\left\{a_{m} t^{\frac{m}{n}}+a_{m-1} t^{\frac{m-1}{n}}+\cdots+a_{1} t^{\frac{1}{n}}+a_{0}:\right. \\
\left.n \in \mathbb{Z}^{>0}, m \in \mathbb{N}, a_{m}, \cdots, a_{1} \in \tilde{\mathbb{Q}}, a_{0} \in \mathbb{Z}, a_{m} \geq 0, m>0 \rightarrow a_{m}>0\right\} .
\end{gathered}
$$

This is equipped with the obvious + and $\cdot$ and the (non-Archimedean and consistent with + and $\cdot$ ) order induced by $t>\mathbb{N}$. We will use Shepherdson's model and also some later results regarding Iop (see, e.g., $[\mathrm{MM}]$ and $[\mathrm{Wi}]$ ) in this paper. Our work continues the study (initiated in $[\mathrm{AM}]$ ) of the fragment Intuitionistic Open Induction, iop, of HA. Some of the results will also be shown to hold for certain stronger fragments of $H A$ as well.

1.3 We adopt the usual Kripke semantics for intuitionistic theories based on $L$. A Kripke structure $\mathcal{K}$ for $L$ has a frame $P$ which is a rooted poset whose partial order is called accessibility. Elements of $P$ are called nodes of $\mathcal{K}$. To each node $\alpha$ of $\mathcal{K}$ is attached a classical structure $M_{\alpha}$ for $L$ in which the interpretation of equality is an $L$-congruence relation which may properly extend the true equality. For any two nodes $\alpha, \beta$, if $\beta$ is accessible from $\alpha$ (that is $\alpha \leq \beta$ ), then the world at $\alpha$ must be a weak substructure of the one at $\beta$. This means $M_{\beta}$ preserves truth in $M_{\alpha}$ of atomic sentences in $L_{\alpha}$ (the extended language obtained by adding new constant symbols for elements of $M_{\alpha}$ ) although tuples of elements of $M_{\alpha}$ may acquire new atomic properties, perhaps equality, in $M_{\beta}$. An atomic $L_{\alpha}$-sentence is forced at $\alpha$ whenever it is satisfied in $M_{\alpha}$. The inductive definitions of forcing for $\vee, \wedge, \exists$ is the same as the corresponding ones for satisfaction or truth in classical structures, while it is stronger for $\rightarrow$ and $\forall$ as it requires the similar classical defining clause to hold at every accessible node. By $\alpha \Vdash \varphi(\bar{x})$, one means $\alpha \Vdash \forall \bar{x} \varphi(\bar{x})$. No node forces absurdity $\perp$, and $\neg A$ is defined as $A \rightarrow \perp$. One says that $\alpha$ decides $\varphi$ whenever $\alpha \Vdash P E M_{\varphi}$. If $\mathcal{K} \Vdash P E M_{\text {atomic }}$, then one can assume that the interpretation of equality in the worlds of $\mathcal{K}$ is the true one and for any two nodes $\alpha \leq \beta, M_{\alpha}$ is a substructure of $M_{\beta}$.

1.4 The instance of the least number principle $L N P$ with respect to a distinguished free variable $x$ on a formula $\varphi(x, \bar{y})$ is the sentence

$$
L_{x} \varphi=L_{x} \varphi(x, \bar{y}): \forall \bar{y}(\exists x \varphi(x, \bar{y}) \rightarrow \exists x(\varphi(x, \bar{y}) \wedge \forall z<x \neg \varphi(z, \bar{y}))) .
$$

Let Lop (resp. lop) denote the classical (resp. intuitionistic) theory axiomatized by $P A^{-}$ together with the scheme $L N P$ restricted to open formulas. ${ }^{1}$

By $I^{t} o p$ (resp. $\left.i^{t} o p\right)$ we mean the classical (resp. intuitionistic) theory based on $P A^{-}$plus the scheme of transfinite induction

$$
I_{x}^{t} \varphi=I_{x}^{t} \varphi(x, \bar{y}): \forall \bar{y}(\forall x(\forall z<x \varphi(z, \bar{y}) \rightarrow \varphi(x, \bar{y})) \rightarrow \forall x \varphi(x, \bar{y}))
$$

for open $\varphi$.

\footnotetext{
${ }^{1}$ Observe that these instances are universal closures of the corresponding ones as appeared in [TD, p.129]. We will be dealing with double negations of instances of the two schemes in sections 3 and 6 , there will be cases where just the weaker doubly negated scheme (the one in which $\neg \neg$ succeeds all $\forall$ 's) is provable.
} 
For a set $\Gamma$ of formulas, notations such as $i \Gamma, I \Gamma, L \Gamma$ and $i^{t} \Gamma$ should now be understood similarly by replacing the class of open formulas by $\Gamma$.

Formula classes $\forall_{1}, \exists_{1}, \Delta_{0}, \Pi_{1}, \Sigma_{1}$ and $\Pi_{2}$ are defined as usual. E.g., by a $\forall_{1}$-formula one means a formula of the form $\forall \bar{x} \varphi(\bar{x}, \bar{y})$ where $\varphi$ is open, while $\Pi_{1}$-formulas have the above form with $\varphi \in \Delta_{0}$. Our use of the word prenex is two-fold, a block of quantifiers followed by either an open or a $\Delta_{0}$-formula, depending on the context.

1.5 For a set of axioms $T$, we denote its classical (resp. intuitionistic) deductive closure by $T^{c}$ (resp. $T^{i}$ ). Every intuitionistic theory (being its own intuitionistic deductive closure) can be written in this form. A $T$-normal Kripke structure means one whose worlds are classical models of $T$. The intuitionistic theory of the class of $T$-normal Kripke structures is denoted $\mathcal{H}(T)$. It was shown in $\left[\mathrm{AM}, 1.2(\mathrm{ii}, \mathrm{iii}), 1.4,2.3\right.$ (ii)] that Kripke models of lop (resp. $\left(P A^{-}\right)^{i}$ ) are precisely the Iop-normal (resp. $P A^{-}$-normal) ones and lop is strictly stronger than iop. Therefore any intuitionistic theory strictly weaker than lop (and in particular $i o p$ ) is sound but not complete with respect to Iop-normal Kripke structures (since the intuitionistic theory which is sound and complete with respect to this class is $\mathcal{H}($ Iop $)=l o p)$. Let $A E O, U A E O$, and $A U E O$ be the sentences $\forall x \exists y(x=2 y \vee x=2 y+1), \neg \neg A E O$, and $\forall x \neg \neg \exists y(x=2 y \vee x=2 y+1)$ respectively. It is also true that no fragment of $i \forall_{1}$ extending $\left(P A^{-}\right)^{i}$ is complete with respect to the class of its end-extension Kripke models. Every end-extension Kripke model of $\left(P A^{-}\right)^{i}$ forces $U A E O \rightarrow A E O$ but, as the proof of [AM 2.3 (ii)] shows, $i \forall_{1} \nvdash U A E O \rightarrow A E O$.

1.6 The set $\mathbb{Z} R$ of axioms for $\mathbb{Z}$-rings is $D O R$ together with the scheme

$$
\forall x \exists y(x=n y \vee x=n y+1 \vee \cdots \vee x=n y+(n-1))
$$

for standard integers $n \geq 2$. Let $\mathbb{Z} R^{+}$be obtained from $\mathbb{Z} R$ by replacing $D O R$ by $P A^{-}$. Clearly $P A^{-}+I_{y}(y z \leq x) \vdash_{c} \forall x \forall z \neq 0 \exists y \exists r(0 \leq r<z \wedge x=y z+r)$ and so $I o p \vdash \mathbb{Z} R^{+}$.

1.7 Let $\neg \neg$ iop denote the intuitionistic theory axiomatized by $\left(P A^{-}\right)^{i}+\left\{\neg \neg I_{x} \varphi: \varphi\right.$ is open $\}$. The theories $\neg \neg i \forall_{1}$ and $\neg \neg l o p$ are defined similarly, by either replacing the class of open formulas by $\forall_{1}$-formulas or the induction scheme by LNP. Also, $\neg \neg i \Pi_{1}$ will stand for the intuitionistic theory axiomatized by $i \Delta_{0}+\left\{\neg \neg I_{x} \varphi: \varphi \in \Pi_{1}\right\}$. For any set $T$ of formulas, we denote $\{\neg \neg \varphi$ : $\varphi \in T\}$ by $U T$.

1.8 We say that $T^{i}$ is closed under Friedman's translation if whenever $T^{i} \vdash \varphi$ and $\psi$ is a formula which has no free variables bound in $\varphi$, then $T^{i} \vdash \varphi^{\psi}$. Here $\varphi^{\psi}$, Friedman's translation of $\varphi$ by $\psi$, is obtained by replacing each atomic subformula $\rho$ of $\varphi$ by $\rho \vee \psi$. It is easy to see that for any axiom $\sigma$ of $P A^{-}$and any formula $\varphi, \sigma \vdash \sigma^{\varphi}$. In particular, $\left(P A^{-}\right)^{i}$ is closed under Friedman's translation. On the other hand $i o p, i \forall_{1}$ and $i \Pi_{1}$ are not, see [AM, 2.3(iii)] and [We2, Cor. 5].

We say that $T^{i}$ is closed under the rule $D N S$ of double negation shift whenever $T^{i} \vdash$ $\forall \bar{x} \neg \neg \varphi(\bar{x})$ implies $T^{i} \vdash \neg \neg \forall \bar{x} \varphi(\bar{x})$. It has the Disjunction Property $D P$ if for all sentences $\phi$ and $\psi, T^{i} \vdash \varphi \vee \psi$ implies $T^{i} \vdash \varphi$ or $T^{i} \vdash \psi$. The theory $T^{i}$ has the property $E D$ of Existential Definability whenever for all formulas $\varphi(x)$ with $T^{i} \vdash \exists x \varphi(x)$, there exists a term $t$ such that $T^{i} \vdash \varphi(t)$. It is closed under the negative translation whenever it proves the negative translation of any formula it proves classically. Recall that the negative translation of a formula is obtained by replacing any subformula of the form $\psi \vee \eta$, resp. $\exists x \psi$, by $\neg(\neg \psi \wedge \neg \eta)$, resp. $\neg \forall x \neg \psi$ and inserting $\neg \neg$ in front of all atomic sub-formulas, except $\perp$. It was shown in [AM, 2.4] that iop 
and $i \forall_{1}$ are closed under the negative translation. Similarly, one can show that $i \Pi_{1}$ is closed under the negative translation. Let us note that $l o p$ is also closed under the negative translation since as observed in [AM, 1.2, 1.4], Iop $\vdash_{c}$ Lop and lop $\vdash_{i}$ iop.

\section{Worlds in Kripke Models of iop}

In this section, we characterize classical structures at the nodes of Kripke models of iop as those models of $P A^{-}$which generate a ring embedable in a $\mathbb{Z}$-ring and construct an $\omega$-framed Kripke model of $i o p$ with no worlds satisfying Iop. We also show that iop has limited prenex or semipositive consequences. A semipositive formula is one all whose implicational subformulas have atomic antecedent.

Proposition 2.1 If $T^{i}$ decides atomic formulas and is closed under the negative translation, then

(i) $T^{c}$ is $\forall_{1}$-conservative over $T^{i}$.

(ii) Each world of any Kripke model of $T^{i}$ can be embedded in a model of $T^{c}$.

(iii) If $T$ is $\exists$-free, then any model of conseq $\forall_{\forall_{1}}\left(T^{c}\right)$ is realizable as a world in some Kripke model of $T^{i}$.

Proof (i) Suppose that $\varphi$ is an open formula and $T^{c} \vdash \forall x \varphi(x)$. Then $T^{i} \vdash(\forall x \varphi(x))^{-}$, that is $T^{i} \vdash \forall x\left(\varphi(x)^{-}\right)$. Now by the easily verified fact that $P E M_{\varphi} \vdash_{i} \varphi^{-} \leftrightarrow \varphi$, we get $T^{i} \vdash \forall x \varphi(x)$.

(ii) By [Ho, Cor. 6.5.3] it is enough to show any such world $D$ is a model of conseq $\forall_{1}\left(T^{c}\right)$. By part (i), any $\forall_{1}$-consequence of $T^{c}$ is provable in $T^{i}$ and is therefore forced at the node corresponding to $D$. Now by decidability of atomic formulas and the formula being prenex, it is satisfied in $D$ (see [Ma, lemma 1(iii)]).

(iii) Any model of conseq $\forall_{1}\left(T^{c}\right)$ is, by [Ho, Cor. 6.5.3] again, embedable in a classical model of $T$. The Kripke model obtained by putting the latter over the former forces $T^{i}$.

Corollary 2.2 Iop is $\forall_{1}$-conservative over iop and $I \forall_{1}$ is $\forall_{1}$-conservative over $i \forall_{1}$.

A similar sort of argument shows that $I \Pi_{1}$ is $\Pi_{1}$-conservative over $i \Pi_{1}$.

Corollary 2.3 For any $M \vDash P A^{-}$, The following are equivalent:

(i) $M$ is a world in a Kripke model of $i o p$.

(ii) $M$ can be embedded in a model of Iop.

(iii) The ring generated by $M$ satisfies classical $\forall_{1}$-consequences of $\mathbb{Z} R$.

(iv) For each prime $p$, there exists a ring-homomorphism from the ring generated by $M$ to the ring of $p$-adic integers.

Proof We know from proposition 2.1 that (i) and (ii) are equivalent. By [MM, 1.4, 1.5, 3A, 3B], parts (ii), (iv) and embedability of the ring generated by $M$ in a $\mathbb{Z}$-ring are equivalent. The latter is, once more by [Ho, Cor. 6.5.3], equivalent to (iii). 
Proposition 2.4 (i) $\mathbb{Z} R \vdash_{c} \forall x, y\left(x^{2}+1 \neq 4 y\right)$.

(ii) $D O R \nvdash_{c} \forall x, y\left(x^{2}+1 \neq 4 y\right)$.

(iii) The model $\mathbb{Z}\left[u, \frac{u^{2}+1}{4}\right] \geq 0$ of $P A^{-}$is not realizable as a world in any Kripke model of iop.

Proof (i) In fact $D O R+A E O$ classically proves the universal formula above.

(ii) Consider the ring $D=\mathbb{Z}\left[u, \frac{u^{2}+1}{4}\right]$ under the order inherited from $\mathbb{Q}[u]$, with $u$ positive and infinitely large. To prove its discreteness, suppose $f(x, y)=\sum_{0 \leq i, j \leq m} a_{i, j} x^{i} y^{j} \in \mathbb{Z}[x, y]$ and $0<f\left(u, \frac{u^{2}+1}{4}\right)<1$. Then $f\left(u, \frac{u^{2}+1}{4}\right.$ ) would be a non-integer (dyadic) rational $r$. Consider the ring homomorphism $e: \mathbb{Q}[u] \rightarrow \mathbb{Q}[\sqrt{3}]$ induced by sending $u$ to $\sqrt{3}$. The restriction of $e$ to $D$ takes values in $\mathbb{Z}[\sqrt{3}]$ and is identity on the Archimedean part of $D$. In particular, $e\left(f\left(u, \frac{u^{2}+1}{4}\right)\right)=e(r)=r$. But this would be a contradiction, since $\mathbb{Z}[\sqrt{3}]$ does not contain any non-integer rational.

(iii) Using corollary 2.3 (equivalence of (i) and (iii)), this is clear from (i) and the proof of (ii) above.

Proposition 2.5 iop $+\mathbb{Z} R^{+} \nvdash_{i}$ lop.

Proof Consider the two-node Kripke model obtained by putting Shepherdson's model above $(t \mathbb{Q}[t]+\mathbb{Z})^{\geq 0}$. This does not force $L_{y}\left(x^{2}<2 y^{2}\right)$.

Proposition 2.6 There exists an $\omega$-framed Kripke model of $i o p$ with no worlds satisfying Iop. ${ }^{2}$

Proof Consider the $\omega$-framed Kripke structure $\mathcal{K}$ with $M_{n}=\left(t^{\frac{1}{n !}} \tilde{\mathbb{Q}}\left[t^{\frac{1}{n !}}\right]+\mathbb{Z}\right)^{\geq 0}$ attached to node $n$. For any $n, M_{n} \vDash P A^{-}$and $t^{\frac{1}{n !+1}} \notin M_{n}$. These imply $M_{n} \not \models I_{x}\left(x^{n !+1} \leq t\right)$ and so $M_{n} \not \models I o p$. Observe that for each $n,(1) M_{n}$ is a substructure of $M_{n+1}\left(\right.$ since $t^{\frac{1}{n !}}=\left(t^{\frac{1}{(n+1) !}}\right)^{n+1} \in$ $\left.M_{n+1}\right),(2) \forall k \leq n:\left(t^{\frac{1}{k}} \tilde{\mathbb{Q}}\left[t^{\frac{1}{k}}\right]+\mathbb{Z}\right)^{\geq 0} \subseteq M_{n}$ and so $(3) \mathcal{S}_{t}(\mathbb{N})=\cup_{n<\omega} M_{n}$. To see $\mathcal{K} \Vdash i o p$, pick an open formula $\varphi(x, \bar{y})$. For $0 \Vdash I_{x} \varphi(x, \bar{y})$ to hold, it suffices (see [AM, 1.1(i), 1.2(iii)]) that for every $n$ and every $\bar{b} \in M_{n}$, there exists $m \geq n$ such that $\forall k \geq m: M_{k}=I_{x} \varphi(x, \bar{b})$. If $\mathcal{S}_{t}(\mathbb{N}) \models$ $\neg \varphi(0, \bar{b}) \vee \forall x \varphi(x, \bar{b})$, then $m=n$ works. Otherwise, consider the least (necessarily nonzero) element $u \in \mathcal{S}_{t}(\mathbb{N})$ such that $\mathcal{S}_{t}(\mathbb{N}) \models \neg \varphi(u, \bar{b})$ and suppose that $l$ is the least nonnegative integer such that $u \in M_{l}$. Then $m=\max \{l, n\}$ works.

Wehmeier proved some limitation on $\Pi_{2}$-consequences of $i \Pi_{1}$ in [We2, thm. 5]. His arguments show that $I \Delta_{0}+\operatorname{conseq}_{\Pi_{1}}\left(I \Pi_{1}\right) \vdash_{c}$ conseq prenex $\left(i \Pi_{1}\right)+\operatorname{conseq}_{\text {semipositive }}\left(i \Pi_{1}\right)$. In the following proposition, we show a similar sort of limitations for iop.

Proposition 2.7 (i) If $\varphi$ is a semipositive or a prenex sentence and $i o p \vdash \varphi$, then $P A^{-}+$ conseq $_{\forall_{1}}\left(\mathbb{Z} R^{+}\right) \vdash_{c} \varphi$.

(ii) conseq $q_{\text {prenex }}($ iop $) \equiv_{c}$ conseq $_{\forall_{2}}($ iop $) \vdash_{c}$ conseq $_{\text {semipositive }}($ iop $)$.

Proof (i) Once again, by [Ho, Cor. 6.5.3], it suffices to show that $\varphi$ is satisfied in any classical model of $P A^{-}$whose generated ring is embedable in a $\mathbb{Z}$-ring. This is clear on the basis of corollary 2.3, [Ma, Lemma 1(iii)] and [We1, Lemma 1.2].

\footnotetext{
${ }^{2}$ On the other hand, it is proved in [We1] that any $\omega$-framed Kripke model of $H A$ is $P A$-normal.
} 
(ii) Clear from (i) and corollary 2.2, since $I o p \vdash \mathbb{Z} R^{+}$and $P A^{-}$is $\forall_{2}$-axiomatized.

If we extend the language by the modified subtraction $\dot{-}$, then replacing the only axiom

$$
\forall x, y \exists z(x \leq y \rightarrow x+z=y)
$$

of $\left(P A^{-}\right)^{i}$ which is not $\forall_{1}$ by

$$
\forall x, y[(x \leq y \rightarrow x+(y \dot{-} x)=y) \wedge(x>y \rightarrow(y \dot{-} x)=0)],
$$

we get a $\forall_{1}$-axiomatized definitional extension of $\left(P A^{-}\right)^{i}$, see [TD, 2.7.2]. Therefore, the sets of universal consequences and of prenex consequences of intuitionistic open induction in this expanded language, will be classically equivalent.

Examples 2.8 (i) We have iop $\nvdash P(2)$, where $P(2)$ is the sentence $\forall u v w \exists x(2 u=v w \rightarrow$ $(2 x=v \vee 2 x=w))$. The reason is that the $\operatorname{ring} \mathbb{Z}[t, \sqrt{2} t]$ can be embedded in a $\mathbb{Z}$-ring, e.g. in $t \mathbb{Q}[\sqrt{2}][t]+\mathbb{Z}$

(ii) Smith asks in [Smi, 5.1] whether Iop proves the first case of Fermat's Last Theorem for exponents $n \geq 3$, that is for a given integer $n \geq 3$, whether $\operatorname{Iop} \vdash^{(?)} 1 F L T(n)$. Here $1 F L T(n)$ is $\forall x y z \exists u\left(x^{n}+y^{n}=z^{n} \rightarrow n u=x y z\right)$. To see that the intuitionistic version of this has a negative answer, put $\mathcal{S}_{t}(\mathbb{N})$ above $\mathbb{Z}[t, \sqrt[n]{2} t] \geq 0$.

\section{3 iop fails the rule $D N S\left(\exists_{1}^{-}\right)$}

In this section, we show that iop is not closed under the rule $D N S\left(\exists_{1}^{-}\right)$of Double Negation Shift for $\exists_{1}$-formulas without parameters and iop $\nvdash \neg \neg l o p$.

Proposition 3.1 There exists a Kripke model of iop on frame $\omega$ which forces $\neg A E O$.

Proof Let $\left(\psi_{n}\right)_{n \in \omega}$ be an enumeration of all open $L$-formulas with a distinguished free variable. Each open formula $\varphi\left(x_{1}, \cdots, x_{k}\right), k \geq 1$, occurs $k$-times in this enumeration.

Put $M_{0}=\mathbb{Z}[t]^{\geq 0}$ and let $\bar{p}_{0,0}, \bar{p}_{0,1}, \cdots$ be a list of all tuples of parameters from $M_{0}$ (an enumeration of $\left.M_{0}^{<\omega}\right)$.

Fix any $k \geq 0$. Assume that for each $i \leq k$ a subsemiring $M_{i}$ of $\mathcal{S}_{t}(\mathbb{N})$ together with an enumeration $\left(\bar{p}_{i, j}\right)_{j \in \omega}$ of $M_{i}^{<\omega}$ is given. For each $0 \leq i, j, m \leq k$ with $i+j \leq k$, if $\bar{p}_{i, j}$ does not have the same arity as the non-distinguished free variables in $\psi_{m}$ or if $\mathcal{S}_{t}(\mathbb{N}) \models \neg \psi_{m}\left(0, \bar{p}_{i, j}\right) \vee$ $\forall x \psi_{m}\left(x, \bar{p}_{i, j}\right)$, where $x$ is the distinguished free variable in $\psi_{m}$, then let $s_{i, j, m}=0$. Otherwise, let $s_{i, j, m}$ be the least element in $\mathcal{S}_{t}(\mathbb{N})$ for which $\mathcal{S}_{t}(\mathbb{N}) \models \neg \psi_{m}\left(s_{i, j, m}+1, \bar{p}_{i, j}\right)$. Let $M_{k+1}=$ $M_{k}\left[s_{i, j, m}: 0 \leq i, j, m \leq k, i+j \leq k\right]^{\geq 0}$.

Consider the Kripke structure on frame $\omega$ with $M_{k}$ attached to node $k$. We want to show that for any $m, 0 \Vdash I_{x} \psi_{m}(x, \bar{y})$. Fix $i \geq 0$ and let $\bar{p}_{i, j} \in M_{i}$, of the same arity as the number of non-distinguished free variables in $\psi_{m}$, be arbitrary. We need to show $i \Vdash I_{x} \psi_{m}\left(x, \bar{p}_{i, j}\right)$. By [AM 1.2(iii), 1.1(i)], it suffices to prove the following claim:

Claim 1 For each $k \geq i+j+m$, we have $M_{k+1} \vDash I_{x} \psi_{m}\left(x, \bar{p}_{i, j}\right)$. 
Proof of Claim 1 The assumption $k \geq i+j+m$ implies that $i+j, m \leq k$. Therefore in constructing $M_{k+1}$ from $M_{k}$, the formula $\psi_{m}\left(x, \bar{p}_{i, j}\right)$ receives attention. If $\mathcal{S}_{t}(\mathbb{N}) \models \neg \psi_{m}\left(0, \bar{p}_{i, j}\right) \vee$ $\forall x \psi_{m}\left(x, \bar{p}_{i, j}\right)$, then $M_{k+1} \models I_{x} \psi_{m}\left(x, \bar{p}_{i, j}\right)$. Otherwise, by construction, the second conjunct of the antecedent of $I_{x} \psi_{m}\left(x, \bar{p}_{i, j}\right)$ fails in $M_{k+1}$ and so $I_{x} \psi_{m}\left(x, \bar{p}_{i, j}\right)$ itself holds there. This establishes claim 1.

Modifying the above Kripke model, we build a slowed-down $\omega$-framed Kripke model of iop for which we verify in claim 2 the existence of infinitely many worlds satisfying $\neg A E O$. Now since the sentence $A E O$ is $\forall_{2}$, that model will force $\neg A E O$ and we will be done with the proposition (as a matter of fact, we will show in proposition 3.4, based on Hilbert's basis theorem, that all the worlds model $\neg A E O)$.

Consider the above construction with the minor modification that each stage is divided into a number of substages, each of which treats just one formula and one tuple (keeping $\omega$ as the index set for stages of construction).

Claim 2 The slowed-down Kripke model has infinitely many worlds classically satisfying $\neg A E O$.

Proof of Claim 2 We inductively define a strictly increasing infinite sequence of nonnegative integers each of which labels a desired world. Let $n_{0}=0$ (observe that $t$ is neither even nor odd in $M_{0}$ ). Assuming $n_{k}$ is defined for some $k \geq 0$, let $n_{k+1}$ be the least positive integer such that $M_{n_{k+1}}=M_{n_{k+1}-1}\left[r t^{\frac{1}{l}}\right]$, where $r \in \tilde{\mathbb{Q}} \backslash\{0\}$ and $l=\operatorname{depth}\left(r t^{\frac{1}{l}}\right)$ is greater than the depth of any element in $M_{n_{k}}$. By the depth of an element in $\mathcal{S}_{t}(\mathbb{N})=\cup_{n \in \mathbb{Z}^{>0}}\left(t^{\frac{1}{n}} \tilde{\mathbb{Q}}\left[t^{\frac{1}{n}}\right]+\mathbb{Z}\right)^{\geq 0}$, we mean the least positive integer $n$ such that the element is in $\left(t^{\frac{1}{n}} \tilde{\mathbb{Q}}\left[t^{\frac{1}{n}}\right]+\mathbb{Z}\right)^{\geq 0}$. So, depth of standard integers is 1 and that of a real algebraic multiple of $t^{\frac{p}{q}}$, where $(p, q)=1$, is $q$. More generally, depth of a finite sum of such terms is the least common multiple of those of its terms. To see that $n_{k+1}$ exists for each $k$, first observe that maxdepth $\left(M_{0}\right)=1$ while if $M_{k+1}=M_{k}[u]$, then $\operatorname{maxdepth}\left(M_{k+1}\right) \leq\left(\operatorname{maxdepth}\left(M_{k}\right)\right)(\operatorname{depth}(u))$. This implies that $\operatorname{maxdepth}\left(M_{n}\right)$ is finite for each $n$. Now, for each $l$ greater than maximum depth of elements of $M_{n_{k}}$, the element $t^{\frac{1}{l}} \in \mathcal{S}_{t}(\mathbb{N})$ enters into a world at some node (consider the formula $\left.x^{l} \leq t\right) .^{3}$

Fix any $k \geq 0$. Assume $M_{n_{k+1}}=M_{n_{k+1}-1}\left[r t^{\frac{1}{l}}\right]$. Obviously, $r t^{\frac{1}{l}}$ is not odd in $M_{n_{k+1}}$. We show that it is not even there either. Suppose not, i.e. assume for the purpose of a contradiction that $\frac{1}{2} r t^{\frac{1}{l}}=f\left(r t^{\frac{1}{l}}\right)$, for some $f(z) \in M_{n_{k+1}-1}[z]$. We must have $\frac{1}{2} r t^{\frac{1}{l}}=g+b r t^{\frac{1}{l}}$, for some $g \in M_{n_{k+1}-1}, b \in \mathbb{Z}$ ( $g$ is the constant term of $f$ and $b$ is the constant term of the coefficient of $z$ in $f(z))$. If $g=0$, then $\frac{1}{2}=b \in \mathbb{Z}$, contradiction. Otherwise, $g$ must be a nonzero real algebraic multiple of $t^{\frac{1}{l}}$, which is again a contradiction.

Theorem 3.2 The theory iop does not validate the rule $D N S\left(\exists_{1}^{-}\right)$.

Proof We know from [AM, 2.3(i)] that iop $\vdash A U E O$, while the above proposition shows iop $\nvdash U A E O$.

\section{Corollary 3.3 iop $\nvdash \neg \neg l o p .{ }^{4}$}

Proof It is easy to see that $P A^{-}+L_{y}(2 y>x) \vdash_{i} A E O$ and so $\neg \neg l o p \vdash U A E O$.

\footnotetext{
${ }^{3}$ This shows that for each $k, M_{k} \not \models I o p$.

${ }^{4}$ On the other hand, $\neg \neg l o p \vdash$ iop (since lop $\vdash$ iop and, as we will see in 4.5 below, $\neg \neg i o p \vdash$ iop).
} 
Proposition 3.4 For each nonstandard $M \models I o p$ including an element $t$ infinitely many times divisible by 2 , there exists an $\omega$-framed Kripke model of iop all whose worlds satisfying $\neg A E O$ and their union is a (countable) model of Iop inside $M$.

Proof Construct a Kripke model as in proposition 3.1 by replacing $\mathcal{S}_{t}(\mathbb{N})$ by $M$. The statement in claim 1 of that proposition shows that the union of the worlds models Iop. ${ }^{5}$ Assume for the purpose of a contradiction that some world models $A E O$. Put $t_{0}=t$ and $t_{l+1}=\frac{t_{l}}{2}$. The ascending chain of ideals $\left(t_{0}\right) \subseteq\left(t_{1}\right) \subseteq\left(t_{2}\right) \subseteq \cdots$ in the ring generated by that model must stop as, by Hilbert's basis theorem, every finitely generated ring is Noetherian. So, for some $n \in \mathbb{N}$ and some $g$ in that world, $0=(2 g-1) t_{n}$. But this is impossible as $2 g-1 \neq 0$ and $t_{n}$ is infinitely large.

\section{Friedman's Translation by Negated Formulas}

In this section, we show that the theories $i o p, i \forall_{1}$, and $i \Pi_{1}$ are closed under some restricted cases of Friedman's translation, most notably (for applications in the next section) by negated formulas.

Proposition 4.1 (i) If $T^{i}$ proves $P E M_{\text {atomic }}$ (resp. $P E M_{\Delta_{0}}$ ) and has a reversely well founded Kripke model not forcing conseq $_{\forall_{2}}\left(T^{c}\right)$ (resp. conseq $_{\Pi_{2}}\left(T^{c}\right)$ ), then $T^{i}$ is not closed under Friedman's translation.

(ii) If $T^{i} \vdash P E M_{\text {atomic }}$, then for all semipositive sentences $\sigma$ and all sentences $\rho, T^{i} \vdash \sigma$ implies $T^{i} \vdash \sigma^{\rho}$.

(iii) The theories $i o p, i \forall_{1}$ and $i \Pi_{1}$ are closed under Friedman's translation by respectively open, $\forall_{1}$, and $\Pi_{1}$-formulas.

Proof (i) From decidability of atomic (resp. $\Delta_{0}$ ) formulas in $T^{i}$ we get, as mentioned in [AM, 2.2], that $T^{c}$ is $\forall_{2}$-conservative (resp. $\Pi_{2}$-conservative) over $\mathcal{H}(T)$. Now observe that a generalization of the arguments for $H A$ in [We1, 9.2] implies that if a fragment $T^{i}$ of $H A$ is closed under Friedman's translation, then every reversely well founded Kripke model of $T^{i}$ forces $\mathcal{H}(T){ }^{6}$

(ii) It suffices to show the following. Let $\psi$ be a sentence and $\mathcal{K}$ a Kripke structure deciding atomic formulas. Then for any semipositive sentence $\varphi$ we have: $\forall \alpha \in \mathcal{K}\left(\alpha \Vdash \varphi \Rightarrow \alpha \Vdash \varphi^{\psi}\right)$. This can be shown by induction on $\varphi$, the less trivial cases in the induction step being the $\rightarrow$ and $\forall$ ones.

$\rightarrow$ : Suppose $\alpha \Vdash \varphi_{1} \rightarrow \varphi_{2}$, where $\varphi_{1}$ is atomic. Let $\beta \geq \alpha$ and $\beta \Vdash \varphi_{1}^{\psi}$. We need to show $\beta \Vdash \varphi_{2}^{\psi}$. If $\beta \Vdash \psi$, then we will be done (since $\psi \vdash_{i} \varphi_{2}^{\psi}$ ). Otherwise, from $\beta \Vdash \varphi_{1} \vee \psi$ we get $\beta \Vdash \varphi_{1}$ and so $\beta \Vdash \varphi_{2}$. Then by induction hypothesis on $\varphi_{2}$, we get the result.

$\forall$ : If $\alpha \Vdash \forall x \varphi(x)$, then $\forall \beta \geq \alpha, \forall b \in M_{\beta}: \beta \Vdash \varphi(b)$. By induction hypothesis, we get

\footnotetext{
${ }^{5}$ When the process is applied to $\mathcal{S}_{t}(\mathbb{N})$ and, as in 3.1 the infinitely large chosen element is the particular one $t$, the union is indeed $\mathcal{S}_{t}(\mathbb{N})$, as this is known to be the minimal model of Iop which includes $t$.

${ }^{6} \mathrm{By}$ [AM, 2.1(iii)], if $T^{i}$ is closed under both Friedman's and the negative translations, then every Kripke model of $T^{i}$ forces $\mathcal{H}(T)$. Here we paid less and got less.
} 
$\beta \Vdash \varphi(b)^{\psi}$ and so $\alpha \Vdash \forall x \varphi(x)^{\psi}$.

(iii) It is straightforward to see that for atomic formulas $\varphi$; arbitrary formulas $\psi, \eta$ and $\theta$; any $* \in\{\wedge, \vee, \rightarrow\}$ and $z \notin\{x, \bar{y}\}$, we have $\forall z\left(I_{x} \varphi(x, \bar{y})\right)^{\psi(z)}=I_{x}(\varphi(x, \bar{y}) \vee \psi(z))$ and $\forall z\left(I_{x}(\eta(x, \bar{y}) * \theta(x, \bar{y}))\right)^{\psi(z)}=I_{x}\left(\eta(x, \bar{y})^{\psi(z)} * \theta(x, \bar{y})^{\psi(z)}\right)$. Now use [DMKV, sec.1, fact (D)] (or lemma 5.1 below) and [TD, exercise 2.1.4].

Note that part (iii) of the above proposition can be considered as a special case of corollary 4.5 below.

Recall the two pruning lemmas in [DMKV]. The first one belongs to general Kripke-model theory. It says that if $\beta$ is a node of a Kripke model $\mathcal{K}, \varphi$ and $\psi$ are formulas in $L_{\beta}$ such that no free variables of $\psi$ are bound in $\varphi$ and $\beta \nVdash \psi$, then $\beta \Vdash \varphi^{\psi}$ iff $\beta \Vdash^{\psi} \varphi$. Here $\Vdash^{\psi}$ denotes forcing in the Kripke structure $\mathcal{K}^{\psi}$ obtained from the original one by pruning away nodes forcing $\psi$.

We say that a fragment $T^{i}$ of $H A$ has the pruning property if whenever $\beta$ is a node of a Kripke model of $T^{i}, \psi \in L_{\beta}$ and $\beta \| \psi$, then $\beta \Vdash^{\psi} T^{i}$. The second pruning lemma in [DMKV] states that $H A$ has this property. As it was proved in [DMKV] for $H A$ itself, a fragment of $H A$ proving $P E M_{\text {atomic }}$ satisfies the pruning property if it is closed under Friedman's translation. Let us note that the converse is also true. Assume that $T^{i}$ has the pruning property and $T^{i} \vdash \varphi$. Then pruning the nodes forcing $\psi$ from a Kripke model of $T^{i}$ not forcing $\psi$ would result in a model of $T^{i}$ and so for any remaining node $\alpha, \alpha \Vdash^{\psi} \varphi$. Therefore, by the first pruning lemma, it must have forced $\varphi^{\psi}$ originally. Note that the mentioned equivalence is indeed true formula by formula (for pruning or translating by).

Lemma 4.2 For any Kripke structure $\mathcal{K}$, any sentence $\sigma$ with $\mathcal{K} \Vdash \sigma$, and any formula $\varphi$, we have $\mathcal{K}^{\neg \varphi} \Vdash \neg \varphi \neg \neg \sigma$.

Proof Suppose $\mathcal{K} \Vdash \sigma(\sigma$ an $L$-sentence $)$ and $\alpha \in \mathcal{K} \neg \varphi$. For any $\beta \geq \alpha$ with $\beta \in \mathcal{K}\urcorner \varphi$, we have $\beta \nVdash \neg \varphi$ and so there exists $\gamma \geq \beta$ such that $\{\delta \in \mathcal{K}: \delta \geq \gamma\} \subseteq \mathcal{K}\urcorner \varphi$. Therefore, from $\gamma \Vdash \sigma$, we get $\gamma \Vdash^{\neg \varphi} \sigma$. This shows $\alpha \Vdash^{\neg \varphi} \neg \neg \sigma$.

Proposition 4.3 $U \operatorname{Th}(\mathbb{N}) \nvdash_{i} P A^{-}$.

Proof Consider the Kripke model obtained by putting a nonstandard model of $\mathrm{Th}(\mathbb{N})$ with an infinitely large positive element $t$ over $\mathbb{N}[t]$. The lower node does not force $\forall x, y \exists z(x \leq y \rightarrow$ $x+z=y)$.

Lemma 4.4 For arbitrary formulas $\varphi$ and $\psi$, we have: $\neg \neg \forall \bar{y}(\varphi(\bar{y}) \rightarrow \forall x \psi(x, \bar{y}))+P E M_{\psi} \vdash_{i}$ $\forall \bar{y}(\varphi(\bar{y}) \rightarrow \forall x \psi(x, \bar{y}))$.

Proof $\neg \neg \forall \bar{y}(\varphi(\bar{y}) \rightarrow \forall x \psi(x, \bar{y})) \vdash_{i} \forall \bar{y} \neg \neg(\varphi(\bar{y}) \rightarrow \forall x \psi(x, \bar{y})) \equiv_{i} \forall \bar{y}(\varphi(\bar{y}) \rightarrow \neg \neg \forall x \psi(x, \bar{y}))$ $\vdash_{i} \forall \bar{y}(\varphi(\bar{y}) \rightarrow \forall x \neg \neg \psi(x, \bar{y}))$.

Corollary 4.5 We have iop $\equiv \neg \neg i o p, i \forall_{1} \equiv \neg \neg i \forall_{1}$, and $i \Pi_{1} \equiv \neg \neg i \Pi_{1}$. These three theories are closed under Friedman's translation by negated formulas.

Proof Observe that each instance of induction in $i o p, i \forall_{1}$ or $i \Pi_{1}$ is of the form present in lemma 4.4 with $\psi$ open or $\Delta_{0}$. Also $\left(P A^{-}\right)^{i} \vdash P E M_{\text {atomic }}$ and $i \Delta_{0} \vdash P E M_{\Delta_{0}}$. Therefore, we have the mentioned equivalences. Now to see their closure under Friedman's translation by negated formulas, first note that if $\mathcal{K} \Vdash$ iop, then by $4.2, \mathcal{K}\urcorner \psi \Vdash \neg \psi$ Uiop and since $\mathcal{K}\urcorner \psi$ is $P A^{-}$normal, $\mathcal{K}^{\neg \psi} \Vdash\left(P A^{-}\right)^{i}$. The proof for $i \forall_{1}$ is similar, while the one for $i \Pi_{1}$ uses the criterion for 
Kripke models of $i \Delta_{0}$ (being $I \Delta_{0}$-normal and $\Delta_{0}$-elementary extension).

\section{Closure under the rules $V R$ and $I P$}

As mentioned above, we know that the theories $i o p, i \forall_{1}$ and $i \Pi_{1}$ are not closed under Friedman's translation. So, it would be of interest to investigate the validity of some of the consequences of closure under Friedman's translation, known to hold say for $H A$, in these cases.

A fragment $T^{i}$ of $H A$ proving $P E M_{\Delta_{0}}$ (resp. $P E M_{\text {atomic }}$ but not $P E M_{\Delta_{0}}$ ) is said to be closed under Visser's Rule $V R$ if whenever it proves $\neg \neg \varphi \rightarrow \varphi$, for a $\Sigma_{1}$-formula (resp. $\exists_{1}$-formula) $\varphi$, then $\varphi$ is decidable in $T^{i}$. It was proved in [DMKV] that $H A$ is closed under $V R$.

The Independence of Premises rule $I P$ is proved for $H A$ in [TD]. This asserts that if $H A \vdash$ $\neg \varphi \rightarrow \exists y \psi$, with $y$ not free in $\varphi$, then $H A \vdash \exists y(\neg \varphi \rightarrow \psi)$. A restricted version where $\varphi$ is a sentence and $\psi$ has only $y$ free is proved in [Dr, P.117]. The latter mentioned proof works for any fragment $i \Gamma$ of $H A$, where $\Gamma$ is a set of formulas. For, each theory $i \Gamma$ has its class of Kripke models closed under Smorynski's operation $\Sigma^{\prime}$, see [Smo]. Besides this restricted $I P$, two further consequences of $\Sigma^{\prime}$-closure are closure under $D P$ and $E D$.

It is also true that the class of Kripke models of lop is closed under $\Sigma^{\prime}$. For, by [AM, proof of 1.4], models of lop are exactly Iop-normal Kripke structures.

Lemma 5.1 For any $T^{i}$ with decidable atomic formulas, any $\exists_{1}$-sentence $\psi$ and arbitrary sentence $\rho$, we have $T^{i} \vdash \psi^{\rho} \leftrightarrow \psi \vee \rho$.

Proof This can be shown by an easy induction on the built-up of $\psi$. Alternatively, one can give a routine model-theoretic proof using the first pruning lemma and [AM, 1.1(ii)].

Theorem 5.2 The theories $i o p, i \forall_{1}$ and $i \Pi_{1}$ are closed under the rules $V R$ and $I P$.

Proof For $V R$, by corollary 4.5 and lemma 5.1, the proof at the end of [DMKV] goes through. For IP, by corollary 4.5 again, the proof on p. 138-139 of [TD] works.

Notice that by $[\mathrm{AM}, 2.1$ (iv)], lop $=\mathcal{H}(I o p)$ is closed under Friedman's translation. Therefore it is also closed under $I P$ and $V R$.

Proposition 5.3 There exist sentences $\eta, \rho$, and $\nu$ such that:

(i) The rule $V R$ is not valid in $i o p+\eta$.

(ii) The rule $D P$ is not valid in $i o p+\rho$.

(iii) The rule $I P$ (even the above restricted version) is not valid in $i o p+\nu$.

Proof In this proof, we denote the sentence $\exists x \exists y\left((x+1)^{2}=2 y^{2}\right)$ by $\operatorname{Rational}(\sqrt{2})$.

(i) Let $\eta$ be $\neg \neg \operatorname{Rational}(\sqrt{2}) \rightarrow \operatorname{Rational}(\sqrt{2})$. The sentence Rational $(\sqrt{2})$ is $\exists_{1}$ and $i o p+\eta \nvdash$ $\operatorname{Rational}(\sqrt{2}) \vee \neg \operatorname{Rational}(\sqrt{2})$ as $\Sigma^{\prime}$ applied to $\mathcal{S}_{t}(\mathbb{N})$ and $\mathbb{N}$ shows. 
(ii) We mention two groups of examples for such $\rho$ 's. Let $\rho=\tau \vee \neg \tau$, where either:

(a) Iop $\vdash \tau$ and iop $\nvdash \neg \neg \tau$ (this happens, e.g., for $\tau=A E O$ as proposition 3.1 shows); or

(b) Iop $\nvdash \tau$ and $I o p \nvdash \neg \tau$ (as it happens, e.g., for $\tau=\operatorname{Rational}(\sqrt{2})$ ).

(iii) Consider $\nu: \neg A E O \rightarrow \operatorname{Rational}(\sqrt{2})$ and the Kripke model $\mathcal{K}$ in the proof of proposition 3.4(ii) where Rational $(\sqrt{2})$ is forced. ${ }^{7}$ Now let $\mathcal{K}_{1}$ be the result of applying $\Sigma^{\prime}$ to $\mathcal{K}$ and $\mathcal{S}_{t}(\mathbb{N})$. It forces $\nu$ but not $\exists y\left(\neg A E O \rightarrow \exists x(x+1)^{2}=2 y^{2}\right)$. To see this, note that for any $l \in \mathbb{N}$, the nodes in $\mathcal{K}_{1}$ forcing $\neg A E O$ are those of $\mathcal{K}$, none of which forces $\exists x(x+1)^{2}=2 l^{2}$.

\section{Some Remarks on $W \neg \neg L N P$ and $I^{t}$}

We have already noticed that with the instances of $L N P$ universally quantified out on the parameters, iop $\nvdash \neg \neg L_{y}(2 y>x)$. On the other hand, we prove in this section that iop is equivalent with the intuitionistic theory axiomatized by $P A^{-}$plus the scheme of weak $\neg \neg L N P$ for open formulas, where universal quantification on the parameters precedes double negation. We also show that $\left(P A^{-}\right)^{i} \vdash l_{1}$ op. Here $l_{1}$ op is the fragment of lop restricting the open least number principle to (open) formulas with just one free variable. We finish the paper by making some remarks on the relation between the schemes $I^{t}$ and $L N P$.

Minimal logic, which appears in the next proposition, is the weakening of intuitionistic logic obtained by dropping the rule $\perp_{i}$ (which allows to conclude any formula from $\perp$, once $\perp$ has been proved with no discharged assumptions), see [TD] and [TS]. By $m$-provability, we mean provability in minimal logic.

Proposition 6.1 If a fragment $i \Gamma$ of $H A$ is $m$-closed under the negative translation and $I \Gamma \vdash$ $L \Gamma$, then for any formula $\varphi(x, \bar{y}) \in \Gamma, i \Gamma \vdash \forall \bar{y} \neg \neg(\exists x \varphi(x, \bar{y}) \rightarrow \exists x(\varphi(x, \bar{y}) \wedge \forall z<x \neg \varphi(z, \bar{y})))$.

Proof The second proof in [TD, p.131] works.

Corollary 6.2 iop $\equiv W \neg \neg l o p$.

Proof To show iop $\vdash W \neg \neg l o p$, it remains to argue that iop is $m$-closed under the negative translation. It is easy to see that each of the following schemes is provable in minimal logic: $A \rightarrow \neg \neg A, \neg(A \vee B) \leftrightarrow \neg A \wedge \neg B, \neg \neg(A \wedge B) \leftrightarrow \neg \neg A \wedge \neg \neg B, \neg \neg(A \rightarrow B) \rightarrow(\neg \neg A \rightarrow \neg \neg B)$, $\neg \neg \forall x A \rightarrow \forall x \neg \neg A, \neg \neg \exists x A \rightarrow \neg \forall x \neg A$. See [TS, p.35] and [Da, p.162] for some of these, where, e.g., the intuitionistic proof in the latter for $\neg \neg A \wedge \neg \neg B \rightarrow \neg \neg(A \wedge B)$ works in minimal logic too. These can be used to show that each axiom of $P A^{-}, m$-proves its negative translation. Furthermore for any formula $\varphi,\left(I_{x}(\varphi)\right)^{-}=I_{x}\left(\varphi^{-}\right)$and if $\varphi$ is open, then so is $\varphi^{-}$.

For the converse, we give a model-theoretic proof. Let $\alpha$ be a node of a Kripke model $\mathcal{K} \Vdash$ $W \neg \neg l o p, \varphi(x, \bar{y})$ an open formula, and $\bar{a} \in M_{\alpha}$ of the same arity as $\bar{y}$. To prove $\alpha \Vdash I_{x} \varphi(x, \bar{a})$, assume without loss of generality that $\alpha \Vdash \varphi(0, \bar{a})$. By lemma 4.4 and [AM, 1.2(iii)], it is enough to show that for every $\beta \geq \alpha$, there exists $\delta \geq \beta$ such that for all $\eta \geq \delta, M_{\eta} \vDash I_{x} \varphi(x, \bar{a})$. Fix $\beta \geq \alpha$. If for all $\gamma \geq \beta, M_{\gamma} \vDash \forall x \varphi(x, \bar{a})$, then we may take $\delta=\beta$. Otherwise, by $\beta \Vdash W \neg \neg l o p$,

\footnotetext{
${ }^{7}$ Note that $\mathcal{K} \Vdash \exists y\left(\neg A E O \rightarrow \exists x(x+1)^{2}=2 y^{2}\right)$ and $\mathcal{K}^{\prime}$, the result of applying Smorynski's '-operation to $\mathcal{K}$, does not force $\nu$.
} 
there will exist $\gamma \geq \beta$ such that $\gamma \Vdash \neg \neg(\exists x \neg \varphi(x, \bar{a}) \wedge \forall z<x \varphi(z, \bar{a}))$. In particular, for some $\delta \geq \gamma$ and some $d \in M_{\delta}, \delta \Vdash \neg \varphi(d, \bar{a}) \wedge \forall z<d \varphi(z, \bar{a})$. Clearly, such a node $\delta$ has the desired property.

From proposition 6.1, one can also conclude that $i \Pi_{1} \vdash W \neg \neg l \Pi_{1}$.

Proposition 6.3 $\left(P A^{-}\right)^{i} \vdash l_{1}$ op.

Proof Take an arbitrary parameter-free open formula $\varphi(x)$. It is easy to see that, over $\left(P A^{-}\right)^{i}$, it is equivalent to a formula of the form $\vee_{i \leq m} \wedge_{j \leq n} P_{i, j}(x) \geq Q_{i, j}(x)$, where all $P_{i, j}(x)$ and $Q_{i, j}(x)^{\prime}$ 's are in $\mathbb{N}[x]$. Let $\mathcal{K}$ be a Kripke model of $\left(P A^{-}\right)^{i}$ and $\alpha$ a node of $\mathcal{K}$ such that $\alpha \Vdash \exists x \vee_{i \leq m} \wedge_{j \leq n} P_{i, j}(x) \geq Q_{i, j}(x)$. Then, for some $a \in M_{\alpha}, M_{\alpha} \vDash \vee_{i \leq m} \wedge_{j \leq n} P_{i, j}(a) \geq Q_{i, j}(a)$. It is enough to show that there exists $q \in \mathbb{N}$ such that $M_{\alpha} \vDash \vee_{i \leq m} \wedge_{j \leq n} P_{i, j}(q) \geq Q_{i, j}(q)$. Suppose not (which implies that $a$ is nonstandard). Then for all $q \in \mathbb{N}$ and $i \leq m$, there exists $j \leq n$ such that $M_{\alpha} \vDash P_{i, j}(q)<Q_{i, j}(q)$. From here, for each $i \leq m$, we see the existence of $j_{i} \leq n$ such that for infinitely many $q \in \mathbb{N}, M_{\alpha} \vDash P_{i, j_{i}}(q)<Q_{i, j_{i}}(q)$. This shows that for each $i \leq m$, the leading coefficient of $P_{i, j_{i}}(x)-Q_{i, j_{i}}(x) \in \mathbb{Z}[x]$ is negative which contradicts $M_{\alpha} \vDash \vee_{i \leq m} \wedge_{j \leq n} P_{i, j}(a) \geq Q_{i, j}(a)$.

Remark. The above proof heavily relies on $\varphi$ being parameter-free. For example, iop $\nvdash$ $\neg \neg L_{y}(2 y>x)$ implies in particular that $\left(P A^{-}\right)^{i} \nvdash L_{y}(2 y-x \geq 0)$. There are suitable parametersubstitutions like $t \in \mathbb{Z}[t]^{\geq 0} \vDash P A^{-}$for $x$ so that the resulting polynomial $2 y-t$ has positive leading coefficient with respect to $y$ but is still negative for all $y \in \mathbb{N}$.

Proposition 6.4 We have iop $\equiv i^{t} o p, i \forall_{1} \equiv i^{t} \forall_{1}$ and $i \Pi_{1} \equiv i^{t} \Pi_{1}$.

Proof First note that, as observed in the proof of [AM, 1.4], Iop $\equiv$ Lop and it follows from our next paragraph below that, for any class $\Gamma$ of formulas closed under $\neg, L \Gamma \equiv I^{t} \Gamma$. Therefore, $I o p \equiv I^{t}$ op. Also, for any $\varphi, I_{x}^{t} \varphi \vdash_{c} I_{x} \varphi$ and if $\Gamma$ is a class of formulas closed under bounded universal quantifications, then $I \Gamma \vdash I^{t} \Gamma$. So, $I \forall_{1} \equiv I^{t} \forall_{1}$ and $I \Pi_{1} \equiv I^{t} \Pi_{1}$. For both directions in each of the three intuitionistic versions, use the corresponding classical equivalence, closure of all theories mentioned above under the negative translation and finally $\left(P A^{-}\right)^{i} \vdash \varphi^{-} \leftrightarrow \varphi$ for open $\varphi$ and the same for $i \Delta_{0}$ and $\Delta_{0}$ formulas.

For every formula $\varphi=\varphi(x, \bar{y})$ in the language of arithmetic or any expansion of it, we have $\vdash_{c} L_{x} \neg \varphi \leftrightarrow I_{x}^{t} \varphi$. In fact, $L_{x} \neg \varphi+P E M_{\varphi} \vdash_{i} I_{x}^{t} \varphi$, since $L_{x} \neg \varphi+P E M_{\varphi} \vdash_{i} \forall \bar{y}(\neg \exists x(\neg \varphi(x, \bar{y}) \wedge$ $\forall z<x \varphi(z, \bar{y})) \rightarrow \neg \exists x \neg \varphi(x, \bar{y})) \vdash_{i} \forall \bar{y}(\forall x \neg(\neg \varphi(x, \bar{y}) \wedge \forall z<x \varphi(z, \bar{y})) \rightarrow \forall x \neg \neg \varphi(x, \bar{y}))$ and $\forall \bar{y}(\forall x \neg(\neg \varphi(x, \bar{y}) \wedge \forall z<x \varphi(z, \bar{y})) \rightarrow \forall x \neg \neg \varphi(x, \bar{y}))+P E M_{\varphi} \vdash_{i} I_{x}^{t} \varphi(x, \bar{y})$. On the other hand, $I_{x}^{t} \varphi+P E M_{\varphi} \nvdash_{i} L_{x} \neg \varphi$. Indeed, $i^{t} o p \equiv_{i}$ iop $\nvdash L_{x} \neg(2 x \leq y)$. Let us observe that there are atomic formulas $\varphi$ in the expansion of the language of arithmetic by a new predicate symbol $R$ such that $L_{x} \neg \varphi \nvdash_{i} I_{x}^{t} \varphi$. To see this, consider the $\omega$-framed Kripke structure for this expanded language, where the $n$th world is the expansion of the $L$-structure $\mathbb{Z}[t] \geq 0$ by interpreting $R$ as $\mathbb{N} \cup\{t-n, t-n+1, \cdots, t\}$. The instance $L_{x}(\neg R(x))$ is forced at every node $n$, since $n \Vdash \neg R(t+1) \wedge \forall x<(t+1) \neg \neg R(x)$. Clearly the root forces $\forall x(\forall z<x R(z) \rightarrow R(x)) \wedge \neg \forall x R(x)$.

\section{References}

[AM] M. Ardeshir and Mojtaba Moniri, Intuitionistic Open Induction and Least Number Prin- 
ciple and the Buss Operator, Notre Dame J. Formal Logic, 39 (1998), 212-220.

[Bu] S. Buss, Intuitionistic Validity in T-normal Kripke Structures, Ann. Pure Appl. Logic, 59 (1993) 159-173.

[Da] D. van Dalen, Logic and Structure, Springer-Verlag, 1997.

[DMKV] D. van Dalen, H. Mulder, E.C. Krabbe, and A. Visser, Finite Kripke Models of HA Are Locally PA, Notre Dame J. Formal Logic, 27 (1986) 528-532.

[Dr] A.G. Dragalin, Mathematical Intuitionism, Introduction to Proof Theory, AMS, 1988.

[Ho] W. Hodges, Model Theory, Cambridge University Press, 1993.

[MM] A. Macintyre and D. Marker, Primes and their Residue Rings in Models of Open Induction, Ann. Pure Appl. Logic, 43 (1989) 57-77.

[Ma] Z. Markovic, On the Structure of Kripke Models of Heyting Arithmetic, Math. Logic Quart. 39 (1993) 531-538.

[Sh] J.C. Shepherdson, A Nonstandard Model for a Free Variable Fragment of Number Theory, Bull. Polish Acad. Sci., 12 (1964) 79-86.

[Smi] S.T. Smith, Fermat's Last Theorem and Bezout's Theorem in GCD Domains, J. Pure Appl. Algebra, 79 (1992) 63-85.

[Smo] C. Smorynski, Applications of Kripke Models, in: Metamathematical Investigations of Intuitionistic Arithmetic and Analysis, A.S. Troelstra (ed), Springer Lecture Notes in Mathematics, V. 344, 1973.

[TD] A.S. Troelstra and D. van Dalen, Constructivism in Mathematics, An Introduction, V.1, North-Holland, 1988.

[TS] A.S. Troelstra and H. Schwichtenberg, Basic Proof Theory, Cambridge University Press, 1996.

[We1] K.F. Wehmeier, Classical and Intuitionistic Models of Arithmetic, Notre Dame J. Formal Logic, 37 (1996) 452-461.

[We2] K.F. Wehmeier, Fragments of $H A$ Based on $\Sigma_{1}$-induction, Arch. Math. Logic, 37 (1997), $37-49$.

[Wi] A.J. Wilkie, Some Results and Problems on Weak Systems of Arithmetic, Logic Colloquium' 77, North-Holland, 1978, 285-296.

School of Mathematics, IPM, P.O.Box 19395-5746, Tehran, Iran. e-mail: ezmoniri@ipm.ir

Mathematics Department, Tarbiat Modarres University, Tehran, Iran, and: School of Mathematics, IPM, P.O.Box 19395-5746, Tehran, Iran e-mail: mojmon@ipm.ir 